\title{
Chiral symmetry and spin dependence of the quark-antiquark forces in quarkonium
}

\author{
J. E. Villate, J. E. Ribeiro, and P. J. de A. Bicudo* \\ Complexo Interdisciplinar II, Avenida Gama Pinto 2, 1699 Lisboa Codex, Portugal \\ D-S. Liu \\ International Center for Theoretical Physics P. O. Box 586, 34100 Trieste, Italy
}

\begin{abstract}
Within the ladder approximation, the general form of the quark-antiquark interaction kernel, consistent with chiral symmetry, is investigated through the combined use of Ward identities for the axial and vector currents. In the charm and bottom sectors we show that chiral symmetry still plays an important role, through the functional form of the quark-antiquark forces. To maintain the chiral symmetry the scalar, pseudoscalar and tensor terms of the local interaction kernel must appear in a combination consistent with the equation $K_{s}=K_{p}=-3 K_{t}$. Within the validity domain of the ladder approximation, this result is independent of the quark current masses. While the vector and axial parts, $K_{v}$ and $K_{a}$, are not constrained by chiral symmetry, they are needed in order to implement spontaneous chiral-symmetry breaking, since the above terms alone would not do. In addition to the usual spin-spin, spin-orbit and tensor interactions, the existence of a Lorentz-tensor term in the kernel gives rise to a second tensor force which does not appear in previous studies of mesonic spectroscopy.
\end{abstract}

PACS numbers: 12.40.Aa, 12.40.Qq, 14.20.Dh, 14.40.Jz

\section{INTRODUCTION}

Due to the non-perturbative nature of strong interactions, inscribed in the non-abelian nature of QCD, the forces among quarks and antiquarks inside hadrons have not yet been derived from basic principles. In spite of this difficulty a lot can still be learned - and in fact has been learned - about bound systems of quarks, through the study of quark phenomenological models inspired in QCD.

Quark potential models postulate that the gluonic degrees of freedom have already been integrated out leaving behind, at least, an effective four-fermi microscopic interaction. The dynamics of fermions, subject to such an effective potential is then thoroughly studied, including the non-perturbative effects arising from fermion condensation. Then, educated guesses for the quark interactions can be used to see whether we can explain the enormous quantity of hadronic experimental data in terms of the same small set of interaction parameters. One of the basic tenets of such guess lies in the Chiral symmetry of strong interactions.

In the case of heavy mesons, even a simple non-relativistic constituent model gives good results for the observed masses. In the case of lighter mesons, chiral models successfully incorporate the results of current algebras and explain a large amount of phenomena related to spontaneous breaking of chiral symmetry [1]. Therefore, a general model of hadronic physics should incorporate - in a natural way - the results both of the quark and chiral models [2]. As in $\mathrm{QCD}$, the interaction among quarks and antiquarks must respect chiral symmetry which is exact except for the presence of quark current mass terms. Furthermore, chiral symmetry must be spontaneously broken to accommodate -in the limit of zero quark masses - for the Nambu-Goldstone pion [3].

A general and elegant approach for mesons consists in solving the Bethe-Salpeter equation for bound states of quarkantiquark [4]. Then the chiral invariance of the microscopic interaction implies the invariance of the Bethe-Salpeter kernel used to describe mesons. The invariance of the kernel can be studied by making use of the Ward identities for the axial and vector currents [5]. Together with the Bethe-Salpeter equations for the corresponding vertex functions, one obtains a gap equation $[2,6]$ and some constraints for the Lorentz structure of the Bethe-Salpeter kernel [7]. At this stage we chose to work in the ladder approximation and, in this approximation, we obtain the constraints on the $q-\bar{q}$ interaction which satisfies these requirements.

Therefore our present study will be concerned with the consequences of this interaction in the spectrum of heavy

*Also atInstituto Superior Tecnico, Avenida Rovisco Pais, 1100 Lisboa Codex, Portugal 
mesons, where this approximation should work well. In the charm and bottom sectors the explicit breaking of the chiral symmetry due to the large quark mass term, overcomes the effect of the chiral condensate on quarks; namely, the constituent masses are very close to the current masses. However, as we will show in this paper, the constraints imposed by chiral symmetry on the nature of the $q \bar{q}$ interaction do not depend on the current quark masses despite that non-zero current quark masses are used in the Ward identities.

It has been argued in the literature that the dominant part of the quark-antiquark confining kernel must be either a Lorentz-vector or -scalar [8]. A purely vector kernel does not reproduce the experimentally obtained spin-orbit splitting of the ${ }^{3} P_{J}$ quarkonium triplet, which is one of the reasons why scalar confinement has been introduced [9]. However, since a purely scalar kernel is not consistent with chiral symmetry, it will be necessary to include also pseudoscalar and tensor terms. Furthermore, either a vector or an axial-vector or eventually a combination of both terms are also needed in order to obtain a gap equation and a mechanism to support spontaneous chiral-symmetry breaking. We consider only the vector term, which seems sufficient to describe the spectrum of heavy mesons.

The paper is divided into six sections and two appendices. We first review briefly the derivation of the gap equation from the Ward identities in Sec. II, extending this study to a general Lorentz structure for the Bethe-Salpeter kernel. Section III contains the derivation of the Salpeter equation for an instantaneous kernel, in a form which makes it easy to relate the bound-state amplitudes to the quantum numbers of the meson. The interaction kernel obtained in the bound state equations of Sec. III is expressed in terms of angular momentum and spin, for the case of a harmonic oscillator potentials, in Sec. IV. The resulting Salpeter equation for the various mesons is given in Sec. V and then solved numerically in Sec. VI. The last Section contains conclusions. Finally, the two appendices give some more details of the calculation leading to the results of Secs. IV and V.

\section{WARD IDENTITIES AND THE GAP EQUATION.}

In this section we derive the BCS gap equation $[2,6]$ for a kernel with a general spin structure, following the Ward-identities approach of Adler and Davis [5].

Let $\widehat{K}\left(p, p^{\prime}, q\right)$ be the Bethe-Salpeter kernel for a system of a quark and an antiquark, where $p$ and $p^{\prime}$ are the incoming momenta and $q$ is the transferred momentum. We will use the ladder approximation, namely that the kernel $\widehat{K}$ depends only on $q$

$$
\widehat{K}\left(p, p^{\prime}, q\right)=K(q) \Omega \otimes \Omega
$$

where $\Omega$ is a four by four matrix and $K(q)$ a function.

The vertex functions, $\Gamma_{\mu}, \Gamma_{\mu}^{5}$ and $\Gamma^{5}$ associated with the vector and axial currents must satisfy a set of Bethe-Salpeter equations [10]

$$
\begin{aligned}
\Gamma_{\mu}\left(p, p^{\prime}\right) & =\gamma_{\mu}+i \int \frac{d^{4} q}{(2 \pi)^{4}} K(q) \Omega S\left(p^{\prime}+q\right) \Gamma_{\mu}\left(p^{\prime}+q, p+q\right) S(p+q) \Omega \\
\Gamma_{\mu}^{5}\left(p, p^{\prime}\right) & =\gamma_{\mu} \gamma_{5}+i \int \frac{d^{4} q}{(2 \pi)^{4}} K(q) \Omega S\left(p^{\prime}+q\right) \Gamma_{\mu}^{5}\left(p^{\prime}+q, p+q\right) S(p+q) \Omega \\
\Gamma^{5}\left(p, p^{\prime}\right) & =\gamma_{5}+i \int \frac{d^{4} q}{(2 \pi)^{4}} K(q) \Omega S(p+q) \Gamma^{5}\left(p^{\prime}+q, p+q\right) S\left(p^{\prime}+q\right) \Omega
\end{aligned}
$$

here the matrix $S$ is the complete quark propagator. The vector Ward identities are

$$
\begin{aligned}
& i\left(p-p^{\prime}\right)^{\mu} \Gamma_{\mu}\left(p^{\prime}, p\right)=S^{-1}\left(p^{\prime}\right)-S^{-1}(p), \\
& i\left(p-p^{\prime}\right)^{\mu} \Gamma_{\mu}^{5}\left(p^{\prime}, p\right)+i 2 m \Gamma^{5}\left(p^{\prime}, p\right)=\gamma_{5} S^{-1}(p)+S^{-1}\left(p^{\prime}\right) \gamma_{5} .
\end{aligned}
$$

Throughout this paper we used the ladder approximation. Consistent with this approximation we discard quark annihilation graphs so that at this stage we do not consider the effects of anomalies in our effective potential model[5]. We now substitute the quark propagator for the self-energy $\Sigma$ using the relation

$$
i S^{-1}(p)=\not p-m-\Sigma(p)
$$

if the vertex functions are eliminated from the Ward identities - for which purpose it is crucial that we have local potentials - using the Bethe-Salpeter equations (2) we obtain

$$
\Sigma(p)=-i \int \frac{d^{4} p^{\prime}}{(2 \pi)^{4}} K\left(p^{\prime}-p\right) \Omega\left(\not p^{\prime}-m-\Sigma\left(p^{\prime}\right)\right)^{-1} \Omega
$$




$$
\begin{aligned}
& =i \int \frac{d^{4} p^{\prime}}{(2 \pi)^{4}} K\left(p^{\prime}-p\right) \gamma_{5} \Omega \gamma_{5}\left(\not p^{\prime}-m-\Sigma\left(p^{\prime}\right)\right)^{-1} \Omega \\
& =i \int \frac{d^{4} p^{\prime}}{(2 \pi)^{4}} K\left(p^{\prime}-p\right) \Omega\left(\not p^{\prime}-m-\Sigma\left(p^{\prime}\right)\right)^{-1} \gamma_{5} \Omega \gamma_{5} .
\end{aligned}
$$

The consistency of these equations constrains the kernel matrix $\Omega$. Let us use the following general form for the self-energy and the kernel matrix

$$
\begin{gathered}
\Sigma(p)=A\left(p^{2}\right)+B\left(p^{2}\right) \not p \\
\widehat{K}=K_{s}+K_{p} \gamma_{5} \otimes \gamma_{5}+K_{v} \gamma_{\mu} \otimes \gamma^{\mu}+K_{a} \gamma_{5} \gamma_{\mu} \otimes \gamma_{5} \gamma^{\mu}+\frac{1}{2} K_{t} \sigma_{\mu, \nu} \otimes \sigma^{\mu, \nu}
\end{gathered}
$$

where the index $(i=s, p, v, a, t)$ in the functions $K_{i}$ stands for scalar, pseudoscalar, vector, axial-vector and tensor respectively, and they are all functions of the four-momentum $q$.

We will work with an instantaneous potential, which implies that $K(q)$ is a function of $\mathbf{q}$ and not of $q_{0}$; and the self-energy (6) then has the form (from now on $p$ will stand for the magnitude of the vector $\mathbf{p}$ and $\widehat{\mathbf{p}}$ will denote the unit vector parallel to $\mathbf{p})$

$$
\Sigma(\mathbf{p})=E(p)[\cos \phi(p)+\sin \phi(p)(\widehat{\mathbf{p}} \cdot \gamma)]-m-\mathbf{p} \cdot \boldsymbol{\gamma},
$$

where $\phi$ and $E$ are two arbitrary functions of $p$. Equation (5) then leads to a set of integral equations for $\phi$ and $E$

$$
\begin{aligned}
& E(p) \cos \phi(p)=m-2 \int d^{3} p^{\prime}\left[K_{v}\left(\mathbf{p}-\mathbf{p}^{\prime}\right)+K_{a}\left(\mathbf{p}-\mathbf{p}^{\prime}\right)\right] \cos \phi\left(p^{\prime}\right) \\
& E(p) \sin \phi(p)=p-\int d^{3} p^{\prime}\left(\widehat{\mathbf{p}} \cdot \widehat{\mathbf{p}}^{\prime}\right)\left[K_{v}\left(\mathbf{p}-\mathbf{p}^{\prime}\right)-K_{a}\left(\mathbf{p}-\mathbf{p}^{\prime}\right)\right] \sin \phi\left(p^{\prime}\right) \\
& \int d^{3} p^{\prime}\left[K_{s}\left(\mathbf{p}-\mathbf{p}^{\prime}\right)+K_{p}\left(\mathbf{p}-\mathbf{p}^{\prime}\right)+6 K_{t}\left(\mathbf{p}-\mathbf{p}^{\prime}\right)\right] \cos \phi\left(p^{\prime}\right)=0 \\
& \int d^{3} p^{\prime}\left[K_{s}\left(\mathbf{p}-\mathbf{p}^{\prime}\right)-K_{p}\left(\mathbf{p}-\mathbf{p}^{\prime}\right)\right]\left(\widehat{\mathbf{p}} \cdot \widehat{\mathbf{p}}^{\prime}\right) \sin \phi\left(p^{\prime}\right)=0
\end{aligned}
$$

The first two integral equations give the solutions for the chiral angle $\phi$ and quark energy $E$, for given vector and axial-vector kernels. If these two kernels are zero, we get the free Dirac field case $\phi=\tan ^{-1}(p / m)$ and $E=\sqrt{p^{2}+m^{2}}$. Non-trivial solutions for various potentials have been given in the literature[2], and have the general behavior of a $\phi$ angle increasing from zero at $p=0$ and approaching $\pi / 2$ at infinity. Now, the third and fourth integral equations should be valid for any value of $\mathbf{p}$; the fact that our kernels depend only on $\mathbf{p}-\mathbf{p}^{\prime}$ together with the functional shape of $\phi$, which gives different weights to different values of the integrating variable $\mathbf{p}^{\prime}$, forces us to conclude that the integrands in these two equations must vanish, leading to

$$
K_{s}(\mathbf{q})=K_{p}(\mathbf{q})=-3 K_{t}(\mathbf{q})
$$

It is important to notice that these constraints are valid only for local potentials [11] and independent of currentquark masses. The vector and axial-vector kernels are not constrained.

With these conditions, the general form of an instantaneous kernel consistent with chiral symmetry is then

$$
\widehat{K}=K_{v}(\mathbf{q}) \gamma_{\mu} \otimes \gamma^{\mu}+K_{a}(\mathbf{q}) \gamma_{\mu} \gamma_{5} \otimes \gamma^{\mu} \gamma_{5}+K_{\chi}(\mathbf{q})\left(1+\gamma_{5} \otimes \gamma_{5}-\frac{1}{6} \sigma_{\mu, \nu} \otimes \sigma^{\mu, \nu}\right)
$$

The function $E$ can be easily eliminated from equations (9) and (10), leading to the so called gap equation for $\phi$ in terms of the kernels $K_{v}$ and $K_{a}$

$$
\begin{aligned}
& m \sin \phi(p)-\int d^{3} p^{\prime} \sin \phi(p) \cos \phi\left(p^{\prime}\right)\left[2 K_{v}(\mathbf{q})+2 K_{a}(\mathbf{q})\right] \\
& =p \cos \phi(p)-\int d^{3} p^{\prime} \cos \phi(p) \sin \phi\left(p^{\prime}\right)\left(\widehat{\mathbf{p}} \cdot \widehat{\mathbf{p}}^{\prime}\right)\left[K_{v}(\mathbf{q})-K_{a}(\mathbf{q})\right] .
\end{aligned}
$$


this equation has also been derived in the context of effective quark Hamiltonians with a vector interaction [2]. As we said above, if both the vector and axial kernels are zero, the solution of the gap equation gives $\phi=\tan { }^{-1}(p / m)$, which coincides with the Foldy-Wouthuysen transformation, $E$ becomes the relativistic energy of a particle of mass $m$ and the quark propagator $S$ equals the Dirac propagator for a free quark. The introduction of a vector or axial kernel gives a "dressed" quark propagator and a quark energy different from $\left(p^{2}-m^{2}\right)^{1 / 2}$. In this case the vacuum is also different from the vacuum of noninteracting quarks and the chiral symmetry is spontaneously broken. The function $\phi$ is called chiral angle.

Once the gap equation is solved, the chiral angle completely defines the quark energy $E$, and the quark propagator and self-energy. Projection operators and a one-body Hamiltonian can be constructed as

$$
\begin{aligned}
\Lambda^{ \pm}(\mathbf{p}) & \equiv \frac{1}{2 E(p)}[E(p) \pm H(\mathbf{p})] \\
H(\mathbf{p}) & \equiv E(p)\left[\cos \phi(p) \gamma_{0}+\sin \phi(p)(\widehat{\mathbf{p}} \cdot \boldsymbol{\alpha})\right] .
\end{aligned}
$$

the quark spinors are then

$$
\begin{aligned}
& u_{s}(\mathbf{p})=[\cos (\phi / 2)-\sin (\phi / 2) \widehat{\mathbf{p}} \cdot \gamma] u_{s}^{0}, \\
& v_{s}(\mathbf{p})=[\cos (\phi / 2)-\sin (\phi / 2) \widehat{\mathbf{p}} \cdot \gamma] v_{s}^{0},
\end{aligned}
$$

where the vectors $u_{s}^{0}$ and $v_{s}^{0}$ are eigenvectors of $\gamma_{0}$ with eigenvalues +1 and -1 . If the vector and axial kernels are zero, the expressions above take the familiar form of the Dirac theory without interaction.

\section{THE SALPETER EQUATION}

In the previous section we saw how the quark dynamical functions can all be obtained as functions of the chiral angle which, in turn, is a solution of the gap equation. The existence and specific form of the solution to the gap equation depend on the interaction kernel $\widehat{K}$. A precise derivation of the kernel from QCD is not possible at present, due to the non-abelian nature of the theory at the energy domain of hadronic physics. Lattice gauge calculations and phenomenological studies of hadron properties have shed some light into the determination of $K$. It is generally believed that it has a one-gluon-exchange short-range part plus a long-range confining term.

The interaction kernel $\widehat{K}$, Eq. (14), can be related to the mesonic mass spectrum via the Salpeter equation. Consider the Bethe-Salpeter equation for the bound state amplitude $\chi(P, p)$ of a quark of mass $m_{1}$ and an antiquark of mass $m_{2}$

$$
\left[\not p_{1}-m_{1}-\Sigma_{1}\left(\mathbf{p}_{1}\right)\right] \chi(P, p)\left[\not p_{2}+m_{2}+\Sigma_{2}\left(-\mathbf{p}_{2}\right)\right]=i \int \frac{d^{4} q}{(2 \pi)^{4}} K(\mathbf{q}) \Omega \chi(P, p-q) \Omega
$$

where $P=p_{1}+p_{2}$ and $p=\left(m_{2} p_{1}-m_{1} p_{2}\right) /\left(m_{1}+m_{2}\right)$ are the total and relative momenta, and $q=p-p^{\prime}$. The indices 1 and 2 label the quark and the antiquark respectively. The integral over $q_{0}$ can be easily done, leading to the Salpeter equations

$$
\begin{aligned}
& \left(E_{1}+E_{2}-M\right) \chi^{+-}=-\Lambda_{1}^{+}(\mathbf{p}) \int \frac{d^{3} p^{\prime}}{(2 \pi)^{3}} K(\mathbf{q}) \gamma_{0} \Omega\left[\chi^{+-}\left(\mathbf{p}^{\prime}\right)+\chi^{-+}\left(\mathbf{p}^{\prime}\right)\right] \Omega \gamma_{0} \Lambda_{2}^{-}(-\mathbf{p}) \\
& \left(E_{1}+E_{2}+M\right) \chi^{-+}=-\Lambda_{1}^{-}(\mathbf{p}) \int \frac{d^{3} p^{\prime}}{(2 \pi)^{3}} K(\mathbf{q}) \gamma_{0} \Omega\left[\chi^{+-}\left(\mathbf{p}^{\prime}\right)+\chi^{-+}\left(\mathbf{p}^{\prime}\right)\right] \Omega \gamma_{0} \Lambda_{2}^{+}(-\mathbf{p}) \\
& \chi^{++}(\mathbf{p})=\chi^{--}(\mathbf{p})=0
\end{aligned}
$$

which have been given in the zero-momentum frame $(\mathbf{P}=0)$, where the meson mass is $M=P_{0}$. The amplitudes $\chi^{ \pm \pm}(\mathbf{p})$ have been defined as

$$
\chi^{ \pm \pm}(\mathbf{p}) \equiv \Lambda_{1}^{ \pm}(\mathbf{p})\left[\int d p_{0} \chi(p)\right] \Lambda_{2}^{ \pm}(-\mathbf{p}) .
$$

It is easier to understand the physical meaning of the terms involved in the Salpeter equation, if we work with two by two matrices whose indices are the spin indices of the quarks, rather than with the four by four matrices $\chi^{ \pm \pm}$. In 
order to do so, we define

$$
\begin{aligned}
& \psi^{+}(\mathbf{p}) \equiv u_{1}^{\dagger}(\mathbf{p}) \chi^{+-}(\mathbf{p}) v_{2}(-\mathbf{p}) \\
& \psi^{-}(\mathbf{p}) \equiv v_{1}^{\dagger}(\mathbf{p}) \chi^{-+}(\mathbf{p}) u_{2}(-\mathbf{p})
\end{aligned}
$$

In terms of these amplitudes, the Salpeter equations reduce to the following system

$$
\left(E_{1}+E_{2} \mp M\right) \psi^{ \pm}+\int d^{3} p^{\prime}\left[\widehat{V}^{ \pm \pm}\left(\mathbf{p}, \mathbf{p}^{\prime}\right) \psi^{ \pm}\left(\mathbf{p}^{\prime}\right)-\widehat{V}^{ \pm \mp}\left(\mathbf{p}, \mathbf{p}^{\prime}\right) \psi^{\mp}\left(\mathbf{p}^{\prime}\right)\right]=0 .
$$

The potentials $\widehat{V}^{ \pm \pm}$and $\widehat{V}^{ \pm \mp}$ are operators in spin and momenta and have been defined as

$$
\begin{aligned}
\widehat{V}^{++}\left(\mathbf{p}, \mathbf{p}^{\prime}\right) & \equiv(2 \pi)^{-3} K\left(\mathbf{p}-\mathbf{p}^{\prime}\right)\left[\bar{u}_{1}(\mathbf{p}) \Omega u_{1}\left(\mathbf{p}^{\prime}\right)\right] \otimes\left[\bar{v}_{2}\left(\mathbf{p}^{\prime}\right) \Omega v_{2}(\mathbf{p})\right]^{T}, \\
\widehat{V}^{--}\left(\mathbf{p}, \mathbf{p}^{\prime}\right) & \equiv(2 \pi)^{-3} K\left(\mathbf{p}-\mathbf{p}^{\prime}\right)\left[\bar{v}_{1}(\mathbf{p}) \Omega v_{1}\left(\mathbf{p}^{\prime}\right)\right] \otimes\left[\bar{u}_{2}\left(\mathbf{p}^{\prime}\right) \Omega u_{2}(\mathbf{p})\right]^{T}, \\
\widehat{V}^{+-}\left(\mathbf{p}, \mathbf{p}^{\prime}\right) & \equiv(2 \pi)^{-3} K\left(\mathbf{p}-\mathbf{p}^{\prime}\right)\left[\bar{u}_{1}(\mathbf{p}) \Omega v_{1}\left(\mathbf{p}^{\prime}\right)\right] \otimes\left[\bar{u}_{2}\left(\mathbf{p}^{\prime}\right) \Omega v_{2}(\mathbf{p})\right]^{T}, \\
\widehat{V}^{-+}\left(\mathbf{p}, \mathbf{p}^{\prime}\right) & \equiv(2 \pi)^{-3} K\left(\mathbf{p}-\mathbf{p}^{\prime}\right)\left[\bar{v}_{1}(\mathbf{p}) \Omega u_{1}\left(\mathbf{p}^{\prime}\right)\right] \otimes\left[\bar{v}_{2}\left(\mathbf{p}^{\prime}\right) \Omega u_{2}(\mathbf{p})\right]^{T} .
\end{aligned}
$$

The expressions inside the square brackets are two by two matrices corresponding to the two spin values of the quark and antiquark; the superscript $T$ stands for matrix transposition. The definition of the potential $\widehat{V}^{++}$coincides with that obtained from the transition matrix $T_{f i}$ for quark-antiquark scattering $[12,13]$. Notice that we do not obtain factors of $\sqrt{E / m}$ due to the normalization that we use for the spinors.

If the positive-energy solution $\psi^{+}$is dominant over the negative-energy solution $\psi^{-}$, the Salpeter equation takes the form of a Schrödinger equation

$$
\left[\left(E_{1}+E_{2}\right) \psi(\mathbf{p})+\int d^{3} p^{\prime} \widehat{V}\left(\mathbf{p}, \mathbf{p}^{\prime}\right) \psi\left(\mathbf{p}^{\prime}\right)\right]=M \psi(\mathbf{p}),
$$

where we have dropped the superscripts. The contraction of the kernel matrices $\Omega$ with the spinors gives several terms; if only the lowest powers of $p$ are kept, one obtains the so-called generalized Breit Hamiltonian [8]. In the case of quarkonium when both the quark and the antiquark have the same large current mass, and the positive-energy channel is certainly dominant over the negative-energy one which can be discarded for all purposes.

\section{SPIN DEPENDENCE OF THE SALPETER INTERACTION KERNEL}

The potential $\widehat{V}$ introduced in the last section have several terms such as spin-spin, spin-orbit and tensor, whose functional form depend upon the interaction kernel $K$. In this paper we restrict our treatment to a quadratic confining potential, which turns the integral Salpeter equations into ordinary differential equations in momentum space. The functions $K_{i}$ in the kernel $\widehat{K}$ then have the form

$$
K_{i}(\mathbf{q})=-\frac{4}{3}(2 \pi)^{3} K_{i}^{3} \nabla^{2} \delta(\mathbf{q})
$$

Introducing this expression into Eq. (14), without an axial-vector term, we have

$$
\widehat{K}(\mathbf{q})=-\frac{4(2 \pi)^{3}}{3}\left[K_{v}^{3} \gamma_{\mu} \otimes \gamma^{\mu}+K_{\chi}^{3}\left(1+\gamma_{5} \otimes \gamma_{5}-\frac{1}{6} \sigma_{\mu, \nu} \otimes \sigma^{\mu, \nu}\right)\right] \nabla^{2} \delta(\mathbf{q}),
$$

where $K_{v}$ and $K_{\chi}$ are two parameters with units of energy, which at this point are arbitrary.

This kernel together with the spinors defined in Eq. (17) can be introduced into Eq.(23) leading to several terms depending on the chiral angle. The details are given in Appendix A and the potential obtained is

$$
\begin{aligned}
\widehat{V}\left(\mathbf{p}, \mathbf{p}^{\prime}\right)= & \delta^{3}\left(\mathbf{p}-\mathbf{p}^{\prime}\right)\left\{-\frac{1}{p^{2}} \frac{\partial}{\partial p}\left[p^{2}\left(f_{S I}+f_{S S} \boldsymbol{\sigma}_{1} \cdot \boldsymbol{\sigma}_{2}+f_{T} \widehat{S}_{12}\right) \frac{\partial}{\partial p}\right]\right. \\
& +\frac{{ }^{2}}{p^{2}}\left[f_{S I}+f_{S S} \boldsymbol{\sigma}_{1} \cdot \boldsymbol{\sigma}_{2}\right]+V_{S I}+V_{S S} \boldsymbol{\sigma}_{1} \cdot \boldsymbol{\sigma}_{2}+V_{S L}\left(\boldsymbol{\sigma}_{1}+\boldsymbol{\sigma}_{2}\right) \cdot \mathbf{L} \\
& \left.+V_{S L}^{\prime}\left(\boldsymbol{\sigma}_{1}-\boldsymbol{\sigma}_{2}\right) \cdot \mathbf{L}+V_{T} \widehat{S}_{12}-\frac{2}{p^{2}} f_{T} \widehat{T}_{12}\right\},
\end{aligned}
$$


where $\sigma_{1}$ and $\sigma_{2}$ are the Pauli matrices acting on spin indices of the quark and antiquark respectively. The operator $\mathbf{L}$ is the angular momentum, while $\widehat{S}_{12}$ and $\widehat{T}_{12}$ are two tensor operators defined as

$$
\begin{gathered}
\widehat{S}_{12} \equiv\left(\boldsymbol{\sigma}_{1} \cdot \widehat{\mathbf{p}}\right)\left(\boldsymbol{\sigma}_{2} \cdot \widehat{\mathbf{p}}\right)-\frac{1}{3}\left(\boldsymbol{\sigma}_{1} \cdot \boldsymbol{\sigma}_{2}\right) \\
\widehat{T}_{12} \equiv \frac{1}{2}\left[\left(\boldsymbol{\sigma}_{1} \cdot \mathbf{p}\right)\left(\boldsymbol{\sigma}_{2} \cdot \nabla_{p}\right)+\left(\boldsymbol{\sigma}_{2} \cdot \mathbf{p}\right)\left(\boldsymbol{\sigma}_{1} \cdot \boldsymbol{\nabla}_{p}\right)\right] \\
-\frac{1}{3}\left(\boldsymbol{\sigma}_{1} \cdot \boldsymbol{\sigma}_{2}\right)\left(\mathbf{p} \cdot \nabla_{p}\right)-\widehat{S}_{12}\left(\mathbf{p} \cdot \nabla_{p}+\frac{1}{2} \widehat{L}^{2}\right)
\end{gathered}
$$

The tensor $\widehat{S}_{12}$ gives rise to the usual tensor force which has been extensively studied. The second tensor, $\widehat{T}_{12}$, has not appeared in earlier studies and arises here due to the tensor term $\sigma_{\mu, \nu} \otimes \sigma^{\mu, \nu}$ included in the Bethe-Salpeter kernel in order to satisfy chiral symmetry; it is a hermitian tensor operator. The anomalous spin-orbit term, $\left(\boldsymbol{\sigma}_{1}-\right.$ $\left.\boldsymbol{\sigma}_{2}\right) \cdot \mathbf{L}$, survives only if the current masses of quark and antiquark are different and it mixes states of different charge conjugation number.

The various terms of the interaction: spin independent $\left(f_{S I}\right.$ and $\left.V_{S I}\right)$, spin-spin $\left(f_{S S}\right.$ and $\left.V_{S S}\right)$, spin-orbit $\left(V_{S L}\right)$, anomalous spin-orbit $\left(V_{S L}^{\prime}\right)$ and tensor $\left(f_{T}\right.$ and $\left.V_{T}\right)$, are all dependent on the chiral angles $\phi_{1}(p), \phi_{2}(p)$, and thus influenced by the chiral condensate. If the quark and the antiquark have the same flavor $($ quarkonium $)$ then $\phi_{1}(p)=$ $\phi_{2}(p)$ and the functions $f$ and $V$ - see Appendix A-simplify considerably. Furthermore, if the quark and antiquark are heavy $-m$ large enough with respect to the potential parameters $K_{v}$ and $K_{\chi}$ - a good approximation to the chiral angle is given by

$$
\phi(p) \approx \tan ^{-1}\left(\frac{p}{m}\right)
$$

This approximation gives the same value of $\phi(p)$ that would be obtained if there were no spontaneous chiralsymmetry breaking. However, the functions $f$ and $V$ have already acquired a functional form determined by the specific form of the interaction kernel consistent with chiral symmetry. As a result, in charmonium and heavier quarkonia chiral symmetry does not manifest itself directly through the chiral angle, but through the spin and angular-momentum dependence of the interaction.

With the above approximation for $\phi$ and keeping only terms up to order $p^{2}$, the functions $f$ and $V$ of Appendix A take a simple form

$$
\begin{aligned}
f_{S I} & =\frac{4}{3}\left(K_{v}^{3}-K_{\chi}^{3}\right)+\frac{4}{3}\left(K_{v}^{3}+K_{\chi}^{3}\right)(p / m)^{2}, \\
f_{S S} & =-\frac{4}{9} K_{\chi}^{3}\left(1+\frac{1}{3}(p / m)^{2}\right), \\
f_{T} & =\frac{8}{9} K_{\chi}^{3}(p / m)^{2} \\
V_{S L} & =2 K_{v}^{3} / m^{2} \\
V_{S I} & =-\frac{8}{3}\left(K_{\chi}^{3} / m^{2}\right), \\
V_{S S} & =\frac{4}{3}\left(K_{v}^{3}-\frac{2}{3} K_{\chi}^{3}\right) / m^{2} \\
V_{T} & =V_{S L}^{\prime}=0 .
\end{aligned}
$$

These are the same expressions that are obtained from the Breit-Fermi Hamiltonian [8, 13], with a quadratic potential and with a Lorentz structure as given by the chiral symmetry. Notice the vanishing of the tensor term $V_{T}$, which is a peculiarity of the quadratic potential.

\section{BOUND STATE EQUATIONS FOR QUARKONIUM}

Now that we know the functional form of the potential, we can write down the bound-state equation for mesons. With Eq. (27), the Hamiltonian becomes

$$
\begin{aligned}
H= & -\frac{1}{p^{2}} \frac{\partial}{\partial p}\left[p^{2}\left(f_{S I}+f_{S S} \boldsymbol{\sigma}_{1} \cdot \boldsymbol{\sigma}_{2}+f_{T} \widehat{S}_{12}\right) \frac{\partial}{\partial p}\right] \\
& +\frac{\widehat{L}^{2}}{p^{2}}\left[f_{S I}+f_{S S} \boldsymbol{\sigma}_{1} \cdot \boldsymbol{\sigma}_{2}\right]+V_{S I}+V_{S S} \boldsymbol{\sigma}_{1} \cdot \boldsymbol{\sigma}_{2}+V_{S L}\left(\boldsymbol{\sigma}_{1}+\boldsymbol{\sigma}_{2}\right) \cdot \mathbf{L} \\
& +V_{S L}^{\prime}\left(\boldsymbol{\sigma}_{1}-\boldsymbol{\sigma}_{2}\right) \cdot \mathbf{L}+V_{T} \widehat{S}_{12}-\frac{2}{p^{2}} f_{T} \widehat{T}_{12}+2 E(p) .
\end{aligned}
$$


Written in this form, each term in $H$ is explicitly hermitian. This is the well-known constituent quark model Hamiltonian. What is new here is that chiral symmetry has determined the functional form of the interaction terms. In particular, the introduction of a scalar plus vector kernel, as favored by phenomenological studies of quarkonium, led to the need for pseudoscalar and tensor terms to maintain the chiral symmetry; the introduction of a tensor term in the kernel gave rise to the tensor interaction $f_{T} \widehat{T}_{12} / p^{2}$ which had not appeared in earlier studies of quarkonium.

The quark energy $E(p)$ also displays the effect of chiral-symmetry breaking through its dependence on the chiral angle $\phi$. Replacing Eq. (30) into Eq. (9) and expanding in powers of $p$, we have

$$
E(p)=m\left[1-8\left(\frac{K_{v}}{m}\right)^{3}\right]+\frac{p^{2}}{2 m}\left[1+\frac{104}{3}\left(\frac{K_{v}}{m}\right)^{3}\right]+\ldots
$$

In addition to the usual terms, we also have chiral contributions of order zero and two. For Charmonium and Bottomonium they are very small.

With the series expansion for $E(p)$ given above and the functions $f$ and $V$ obtained in the previous section, the functional form of the Hamiltonian $H$ is determined in terms of two parameters $K_{v}^{3}$ and $K_{\chi}^{3}$. The angular part of the wave function $\psi$ is given by a spherical harmonic of angular momentum $\mathbf{L}$, which couples to a spherical harmonic of spin $S$, to give a total angular momentum $J$. The equations obtained for the radial part of the wave function are

$$
\begin{aligned}
& -\frac{1}{p^{2}} \frac{d}{d p}\left[\left(A_{S} p^{2}+B_{J L} p^{4}\right) \frac{d \psi_{J L S}}{d p}+C_{J L} p^{4} \frac{d \psi_{J, 2 J-L, S}}{d p}\right]+\left[\frac{L(L+1)}{p^{2}} A_{S}\right. \\
& \left.+D p^{2}+E_{J L S}\right] \psi_{J L S}+(J-1)(J+2) C_{J L} \psi_{J, 2 J-L, S}=\left(M_{J L S}-2 m\right) \psi_{J L S},
\end{aligned}
$$

where the eigenvalue $M_{J L S}$ is the meson mass, and $m$ is the current-quark mass. The constants $A_{S}, B_{J L}, C_{J L}, D$ and $E_{J L S}$ are given in Appendix B. For a given positive value of $J$, the spin $S$ can only take values of 0 or 1 . In the case $S=0$ we have $L=J$, the constant $C_{J J}$ vanishes and there is only one differential equation for $\psi_{J J}$. While for $S=1$ the orbital angular momentum takes two values $L=J \pm 1$ and there are two coupled equations for the two components of the wave function: $\psi_{J, J-1,1}$ and $\psi_{J, J+1,1}$. The case $J=0$ gives rise to two equations without coupling: one for the $\eta$ meson $\left(\psi_{000}\right)$, and the other for the $\chi_{0}$ meson $\left(\psi_{011}\right)$.

\section{NUMERICAL SOLUTION OF THE BOUND STATE EQUATIONS AND DISCUSSION OF THE RESULTS}

The bound state equations of quarkonium derived in the previous section have been solved numerically using the method of relaxation. The procedure we have used is the following: For charmonium we first choose arbitrary values of the potential-strength $K_{\chi}$ and the quark mass $m$, given as ratios to the vector potential-strength $K_{V}$. We then find the complete mass spectrum by solving the Salpeter equations for the various combinations of quantum numbers with $J$ up to 2 . We obtain the value of $K_{V}$ that minimizes the errors of the known meson masses. The procedure is then repeated for different values of $m$ and $K_{\chi}$ and we look for a set of values that gives the best results.

In the case of bottomonium, the potential-strengths $K_{\chi}$ and $K_{v}$ are already given by the charmonium results, and one is left to adjust the mass $m_{b}$ to attempt a good agreement with the experimental values. In tables I and II we present the results of our study. The set of parameters which gave the best results as explained above, was the following:

$$
\begin{aligned}
& m_{c}=1330 \mathrm{MeV} \\
& m_{b}=4730 \mathrm{MeV} \\
& K_{v}=281.7 \mathrm{MeV} \\
& K_{\chi}=-136.0 \mathrm{MeV} .
\end{aligned}
$$

Overall, the results of tables I and II are quite reasonable and constitute already a substantial improvement over previous results obtained within this model [15], despite the fact we have not yet included the vectorial Coulomb force. But rather than attempting here a detailed phenomenological description of the data, what we wanted was to illustrate some new striking - and strong - effects of chiral symmetry which remain operational even in the heavy quark sector.

We have shown that if we want to respect Chiral symmetry then, whenever we add a scalar component to the $q-\bar{q}$ kernel, an extra special combination of a tensorial plus a pseudo-scalar potential must also be introduced. It turns out that together with the vectorial confining force, the inclusion, in our $q-\bar{q}$ kernel, of such a combination 
improves substantially the overall results, both for charmonium and bottomonium, over those obtained with a pure Lorentz-vector confining force [15].

A complete study which should include not only the confining force but also the Coulomb force is postponed for a future paper. The introduction of a potential going like $1 / r$ would transform our already complicated, but still manageable differential Salpeter equations, into integral equations thereby loosing the mathematical simplicity of the model. The same could be said for a linearly rising potential.

That we are going to need a Coulomb force is immediately seen by the analysis of the mass splittings of the ${ }^{3} P_{J}$ triplet,

$$
R \equiv \frac{M\left(2^{++}\right)-M\left(1^{++}\right)}{M\left(1^{++}\right)-M\left(0^{++}\right)}
$$

We get $R \approx 2.5$ (instead of the experimental values of 0.48 and 0.66 for charmonium and bottomonium respectively) which is an indication that we need Coulomb forces.

The interplay of a scalar confining potential and the Coulomb vectorial potential can be used to reproduce the experimental values of $R$. With only the scalar term, $R$ takes a value of 2 ; but the addition of the Coulomb term brings $R$ down to a value lower than $0.8[8,9]$. In our case, because we also have a scalar term, we expect a similar thing to happen. The difference is that a pure scalar confining potential does not respect chiral symmetry and will prove inadequate for light mesons whereas our $q-\bar{q}$ kernel satisfies this symmetry and therefore supports the pion as a pseudo Nambu-Goldstone boson.

The spatial dependence of the potential such as given in Eq.(14) must also reflect the effect of coupled channels to the mesonic masses. It is known [16] that coupled channels contribute in a sizable way to the masses of mesons, the effect being larger for the ground state. Once integrated out, the effect of these coupled channels can be simulated by an "effective-optical" potential less steeper than the microscopic potential we started with. However, an explicit and self consistent calculation of the coupled channels contribution for charmonium and bottomonium is also beyond the scope of this work.

\section{CONCLUSIONS}

We have studied, in the ladder approximation, the consequences of chiral symmetry in the functional form of the mesonic Bethe-Salpeter kernel. As a result, if the $q-\bar{q}$ kernel has a scalar part, it must also have -in order to maintain chiral symmetry up to current-quark-mass breaking terms - a pseudoscalar and tensor parts in a special combination. This constraint on the possible combinations of scalar, pseudoscalar and tensorial forces is valid for any local potential and does not depend on the current quark masses; it leads to a specific spin dependence of the interactions. In particular, the introduction of a tensor kernel leads to a second tensor force which has not appeared in earlier studies.

Although affecting in general the hadronic spectra, the chiral constraint on the interaction kernel decouples from the mass gap equation and thence will not affect the pion. Another consequence is that we still need a vectorial confining term (with or without an axial-vector partner) to induce spontaneous chiral-symmetry breaking which is a prerequisite to address the phenomenology of the light meson sector. Together with a Lorentz-vectorial confining force we have tested the chirally-invariant combination of scalar, pseudoscalar and tensorial forces both in the charmonium and bottomonium sectors. Overall, the results are good and greatly improved over those using a purely vectorial confining force. However the R-analysis of the ${ }^{3} P_{J}$ points out the need to introduce Coulomb forces to explain these splittings.

\section{APPENDIX A}

In this appendix we will derive the expression for the potential $\widehat{V}$ given in Eq. (27). We can rewrite the kernel (26) in a form which will be more convenient for our calculations

$$
\begin{aligned}
\widehat{K}(\mathbf{q})=-\frac{4(2 \pi)^{3}}{3} \quad & {\left[K_{v}^{3}\left(\gamma_{0} \otimes \gamma_{0}-\gamma_{j} \otimes \gamma_{j}\right)\right.} \\
& \left.+K_{\chi}^{3}\left(1+\gamma_{5} \otimes \gamma_{5}-\frac{1}{3} \alpha_{j} \otimes \alpha_{j}-\frac{1}{3} \Sigma_{j} \otimes \Sigma_{j}\right)\right] \nabla^{2} \delta(\mathbf{q}) .
\end{aligned}
$$

Next, we notice that the spinors $v$ in the potential $\widehat{V}^{++}$can be replaced by $u$-spinors using the relation

$$
\left[\bar{v}\left(\mathbf{p}^{\prime}\right) \Gamma v(\mathbf{p})\right]^{T}= \pm \bar{u}(\mathbf{p}) \Gamma u\left(\mathbf{p}^{\prime}\right)
$$


where the plus sign holds for $\Gamma=\gamma_{0}, \gamma_{5}$ or $\Sigma_{j}$, and the minus sign for $\Gamma=1, \gamma_{j}$ or $\alpha_{j}$. With this relation and using equations (23) and (25), the potential becomes

$$
\widehat{V}=-\frac{4}{3}\left\{K_{v}^{3}\left[f\left(\gamma_{0}\right)+f(\boldsymbol{\gamma})\right]+K_{\chi}^{3}\left[f\left(\gamma_{5}\right)-f(1)+\frac{f(\boldsymbol{\alpha})}{3}-\frac{f(\boldsymbol{\Sigma})}{3}\right]\right\} \nabla^{2} \delta(\mathbf{q})
$$

where the function $f$ has been defined as

$$
f(\boldsymbol{\Gamma}, \mathbf{p}, \mathbf{q}) \equiv \bar{u}_{1}(\mathbf{p}) \Gamma_{j} u_{1}(\mathbf{p}-\mathbf{q}) \bar{u}_{2}(\mathbf{p}) \Gamma_{j} u_{2}(\mathbf{p}-\mathbf{q}) .
$$

Each of the terms in the potential leads to an integral in the Salpeter equation, which can be evaluated in following way

$$
\begin{array}{ll}
\int d^{3} q \quad & \nabla^{2} \delta(\mathbf{q}) f(\Gamma, \mathbf{p}, \mathbf{q}) \psi(\mathbf{p}-\mathbf{q})=2\left[\mathbf{H}_{1}(\Gamma) F_{2}(\Gamma)+F_{1}(\Gamma) \mathbf{H}_{2}(\Gamma)\right] \cdot \boldsymbol{\nabla} \psi \\
& F_{1}(\Gamma) F_{2}(\Gamma) \nabla^{2} \psi+\left[F_{1}(\Gamma) G_{2}(\Gamma)+G_{1}(\Gamma) F_{2}(\Gamma)+2 \mathbf{H}_{1}(\Gamma) \cdot \mathbf{H}_{2}(\Gamma)\right] \psi
\end{array}
$$

The functions $F, G$ and $\mathbf{H}$ above stand for

$$
\begin{aligned}
F_{i}(\Gamma, \mathbf{p}) & \equiv \bar{u}_{i}(\mathbf{p}) \Gamma u_{i}(\mathbf{p}), \\
G_{i}(\Gamma, \mathbf{p}) & \equiv \bar{u}_{i}(\mathbf{p}) \Gamma \nabla^{2} u_{i}(\mathbf{p}), \\
\mathbf{H}_{i}(\Gamma, \mathbf{p}) & \equiv \bar{u}_{i}(\mathbf{p}) \Gamma \nabla u_{i}(\mathbf{p}) ;
\end{aligned}
$$

they can be evaluated using the definition of the spinors $u$, in terms of the chiral angle, as given in Sec. III. The results are shown in tables III through V. With the help of these tables, and using Eq. (A5), the potential can be written in the form given in Eq. (27), where the various functions have the following functional form (the symbols $c$, $d, s$ and $t$ are defined in table IV)

$$
\begin{aligned}
f_{S I}= & -\frac{4}{3} K_{\chi}^{3} c_{1} c_{2}+\frac{4}{3} K_{v}^{3}\left(1+s_{1} s_{2}\right), \\
f_{S S}= & -\frac{4}{27} K_{\chi}^{3}\left(c_{1} c_{2}+2 s_{1} s_{2}+2\right), \\
f_{T}= & \frac{4}{9} K_{\chi}^{3}\left(1-c_{1} c_{2}+s_{1} s_{2}\right), \\
V_{S I}= & -\frac{2}{3} K_{\chi}^{3}\left[2 t_{1}^{2} c_{1} c_{2}+2 t_{1} t_{2}\left(\frac{1}{3}-s_{1} s_{2}\right)+2 d_{1} s_{1} c_{2}\right. \\
& \left.+\frac{1}{3}\left(7 c_{1} c_{2}-8 c_{1}+s_{1} s_{2}+1\right) p^{-2}\right]-\frac{2}{3} K_{v}^{3}\left[-2 t_{1}^{2}\left(s_{1} s_{2}+1\right)\right. \\
& \left.+2 t_{1} t_{2} c_{1} c_{2}+2 d_{1} c_{1} s_{2}+\left(2 c_{1}-s_{1} s_{2}-2\right) p^{-2}\right]+(1 \leftrightarrow 2), \\
V_{S L}^{\prime}= & -\left(\frac{8}{9} K_{\chi}^{3}+\frac{2}{3} K_{v}^{3}\right) c_{1} p^{-2}-(1 \leftrightarrow 2), \\
V_{S L}= & \frac{2}{9} K_{\chi}^{3} p^{-2}\left(4 c_{1}-s_{1} s_{2}-3 c_{1} c_{2}-1\right)+\frac{2}{3} K_{v}^{3} p^{-2}\left(1-c_{1}+s_{1} s_{2}\right)+(1 \leftrightarrow 2), \\
V_{S S}= & -\frac{2}{27} K_{\chi}^{3}\left[2 t_{1}^{2}\left(c_{1} c_{2}+2 s_{1} s_{2}+2\right)-2 t_{1} t_{2}\left(2 c_{1} c_{2}+s_{1} s_{2}-3\right)\right. \\
& \left.+\left(2 d_{1}-4 d_{2}\right) s_{1} c_{2}+\left(3 c_{1} c_{2}-8 c_{1}+5 s_{1} s_{2}+5\right) p^{-2}\right] \\
& +\frac{2}{9} K_{v}^{3}\left[4 t_{1} t_{2}+\left(c_{1} c_{2}-2 c_{1}+2 s_{1} s_{2}+1\right) p^{-2}\right]+(1 \leftrightarrow 2), \\
V_{T}= & -\frac{2}{9} K_{\chi}^{3}\left[2 t_{1}^{2}\left(c_{1} c_{2}-s_{1} s_{2}-1\right)+2 t_{1} t_{2}\left(c_{1} c_{2}-s_{1} s_{2}+3\right)+2\left(d_{1}+d_{2}\right) c_{1} s_{2}\right. \\
& \left.+4\left(c_{1}-s_{1} s_{2}-1\right) p^{-2}\right]-\frac{1}{3} K_{v}^{3}\left[4 t_{1} t_{2}+\left(c_{1} c_{2}-2 c_{1}-s_{1} s_{2}+1\right) p^{-2}\right] \\
& +(1 \leftrightarrow 2) .
\end{aligned}
$$

\section{APPENDIX B}

We will derive here the differential equation (34). When the spherical tensors in the Hamiltonian (32) act on the radial functions $\psi_{J L S}$, the result is a superposition of functions $\psi_{J^{\prime} L^{\prime} S^{\prime}}$ which can be calculated with standard 
techniques of spherical-tensor algebra. For the spin-spin, spin-orbit and tensor terms we have

$$
\begin{aligned}
& \left(\boldsymbol{\sigma}_{1} \cdot \boldsymbol{\sigma}_{2}\right) \psi_{J L S}=(4 S-3) \psi_{J L S}, \\
& \left(\boldsymbol{\sigma}_{1}+\boldsymbol{\sigma}_{2}\right) \cdot \mathbf{L} \psi_{J L S}=[J(J+1)-L(L+1)-2 S] \psi_{J L S}, \\
& \widehat{S}_{12} \psi_{J L S}=\frac{S\left(L-J-\delta_{J, L}\right)[J(J+1)-L(L+1)-2 S]}{3\left(2 J+1-2 \delta_{J, L} J\right)} \psi_{J L S} \\
& +\frac{2\left(1-\delta_{J, L}\right) \sqrt{J(J+1)}}{2 J+1} \psi_{J, 2 J-L, S} .
\end{aligned}
$$

To calculate the result for the tensor $\widehat{T}_{12}$ we first write it in the form

$$
\widehat{T}_{12}=p \mathrm{~T}_{2}\left(\boldsymbol{\sigma}_{1}, \boldsymbol{\sigma}_{2}\right) \cdot \mathrm{T}_{2}(\widehat{\mathbf{p}}, \boldsymbol{\nabla})-\widehat{S}_{12}\left(p \frac{\partial}{\partial p}+\frac{\widehat{L}^{2}}{2}\right)
$$

where $\mathrm{T}_{2}$ stands for the second-rank tensor obtained from coupling its two arguments [17]. Making use of the so-called gradient formula, one can calculate the reduced matrix element of the tensor $\mathrm{T}_{2}(\widehat{\mathbf{p}}, \boldsymbol{\nabla})$

$$
\begin{aligned}
& \left(L\left\|\mathrm{~T}_{2}(\widehat{\mathbf{p}}, \boldsymbol{\nabla})\right\| L^{\prime}\right)= \\
& \sqrt{\frac{5}{(2 L+1)}}\left\{\begin{array}{ccc}
L^{\prime} & L & 2 \\
1 & 1 & L^{\prime}+1
\end{array}\right\}\left[\delta_{L, L^{\prime}+2} \sqrt{L(L-1)}-\delta_{L, L^{\prime}}(L+1)\right]\left(\frac{\partial}{\partial p}-\frac{L^{\prime}}{p}\right) \\
& +\sqrt{\frac{5}{(2 L+1)}}\left\{\begin{array}{ccc}
L^{\prime} & L & 2 \\
1 & 1 & L^{\prime}-1
\end{array}\right\}\left[\delta_{L, L^{\prime}-2} \sqrt{L^{\prime}\left(L^{\prime}-1\right)}-\delta_{L, L^{\prime}} L\right]\left(\frac{\partial}{\partial p}+\frac{L^{\prime}+1}{p}\right) .
\end{aligned}
$$

For the reduced matrix elements of $\mathrm{T}_{2}\left(\boldsymbol{\sigma}_{1}, \boldsymbol{\sigma}_{2}\right)$ we have

$$
\left(S\left\|\mathrm{~T}_{2}\left(\boldsymbol{\sigma}_{1}, \boldsymbol{\sigma}_{2}\right)\right\| S^{\prime}\right)=\delta_{S, S^{\prime}} \delta_{S, 1} \sqrt{\frac{20}{3}} .
$$

With these last two results and using the tables for the $6 j$ symbols [17], we obtain

$$
\begin{aligned}
\widehat{T}_{12} \psi_{J L S}= & \frac{S\left(L-J-\delta_{J, L}\right)[J(J+1)-L(L+1)-2 S][3-L(L+1)]}{6\left(2 J+1-2 \delta_{J, L} J\right)} \psi_{J L S} \\
& +\frac{\left(\delta_{J, L}-1\right)(J-1)(J+2) \sqrt{J(J+1)}}{2 J+1} \psi_{J, 2 J-L, S} .
\end{aligned}
$$

The eigenvalue equation for the Hamiltonian (32), with the expressions for the functions $f$ and $V$ for quarkonia given in Sec. IV, and the quark energy (33), becomes the ordinary differential equation (34), with the coefficients $A$, $B, C, D$ and $E$ given by

$$
\begin{aligned}
A_{S}= & \frac{4}{3} K_{v}^{3}-\frac{16}{9} S K_{\chi}^{3}, \\
B_{J L}= & \frac{4 K_{v}^{3}}{3 m^{2}}+\frac{16 K_{\chi}^{3}}{9 m^{2}}\left[\frac{2 J(J+1)\left(1+\delta_{J, L}\right)+\delta_{J, L}}{(2 J+1)(J+L+1)}\right], \\
C_{J L}= & \frac{16 K_{\chi}^{3}}{9 m^{2}}\left(1-\delta_{J, L}\right) \frac{\sqrt{J(J+1)}}{(2 J+1)}, \\
D=\frac{1}{m} & +\frac{104 K_{v}^{3}}{3 m^{4}}, \\
E_{J L S}= & \frac{2 K_{v}^{3}}{3 m^{2}}[2 S-30-L(L+1)+3 J(J+1)] \\
& +\frac{16 K_{\chi}^{3}}{9 m^{2}}\left\{\frac{J(J+1)\left[2 L(L+1)\left(1+\delta_{J, L}\right)-6+7 \delta_{J, L}\right]}{(2 J+1)(J+L+1)}-3 S \delta_{J, L}\right\} .
\end{aligned}
$$


[1] A very extensive study appeared recently in: A. Barducci, R. Casalbouni, S. de Curtis, D. Dominici and R. Gatto, Phys. Rev. D 38, 238 (1988).

[2] M. Finger, D. Horn, and J. E. Mandula, Phys. Rev D 20, 3253 (1979); A. Le Yaouanc, L. Oliver, S. Ono, O. Pène and J-C. Raynal, Phys.Rev. D 31, 137 (1985); P. J. de A. Bicudo, J.E.F.T. Ribeiro Phys. Rev. D 42, 1611 (1991); A recent reference is: J.-F. Lagäe, Phys. Rev. D 45, 317(1992).

[3] The first model explicitly displaying spontaneous breaking of chiral symmetry was proposed, already back in the sixties, in the context of the Nucleon-Nucleon interaction by Y. Nambu and G. Jona-Lasinio using analogies with the BCS theory of superconductivity Y. Nambu and G. Jona-Lasinio Phys. Rev. D 122, 345 (1961). See also Ref[6].

[4] Among the many references in the Bethe-Salpeter equation we will just mention two review papers: N. Nakanashi, Supp, Prog. Theor. Phys. 43, 1,(1969); C. H. Llewellyn Smith, Ann. Phys. 53, 521 (1969). For a recent review of the Salpeter equation applied to bound-states of quarks see Ref. [13].

[5] S. L. Adler and A. C. Davis, Nucl. Phys. B 244, 469 (1984).

[6] For a good reference on the mass-gap equation in the BCS theory of Superconductivity see J. R. Schrieffer, Theory of Superconductivity (W. A. Benjamin, 1968).

[7] An early attempt in this direction, using also the Ward identities, was done by Y. Dai, C. Huang and D. Liu, Phys. Rev. D 43, 1717 (1991).

[8] D. Gromes, Nucl. Phys. B 207, 80 (1977).

[9] A. B. Henriques, B. H. Kellet and R. G. Moorhouse, Phys. Lett. 64B, 85 (1976).

[10] In this paper we use the same notation and metric as in: C. Itzykson and J.-B. Zuber, Quantum Field Theory (Mc Graw-Hill, New York, 1980).

[11] If the kernel did not depend only on $p-p^{\prime}$, but rather on $p$ and $p^{\prime}$ separately, rendering it non-local, then it would be possible to relax the above constraint as done by F. Gross and J. Milana, Phys. Rev. D43, 2401 (1991).

[12] T. Barnes and G. I. Ghandour, Phys. Lett. 118B, 411(1982).

[13] W. Lucha, F. F. Schöberl and D. Gromes, Phys. Rep. 200, 127 (1991).

[14] Particle Data Group, J. J. Hernández et al., Phys. Lett. 239B. 1 (1990).

[15] P.J.A. Bicudo, G. Krein, J. E. F. T. Ribeiro and J. E. Villate, Phys. Rev. D 45, 1673 (1992).

[16] E. van Beveren, C. Dullemond and G. Rupp, Phys. Rev. D 21, 105 (1980); E. van Beveren, C. Dullemond and G. Rupp, Phys. Rev. D 27, 1527 (1983); P. J. de A. Bicudo, J.E.F.T. Ribeiro Phys. Rev. D 42, 1635 (1991).

[17] In the definition of coupling of tensors and reduced matrix elements we follow the conventions of: D. M. Brink and G. R. Satchler, Angular Momentum (Clarendon Press, 2nd edit., Oxford, 1968).

TABLE I: Charmonium mass-spectrum in units of $\mathrm{GeV}$, obtained as explained in the text. The experimental values are from Ref. [13].

\begin{tabular}{lllll}
\hline \hline Meson & $\mathrm{J}^{\mathrm{PC}}$ & ${ }^{\mathrm{S}} \mathrm{L}_{\mathrm{J}}$ & Theory & Experiment \\
\hline$\eta_{c}$ & $0^{-+}$ & ${ }^{1} S_{0}$ & 2.978 & 3.979 \\
$J / \psi$ & $1^{--}$ & ${ }^{3} S_{1}+{ }^{3} D_{1}$ & 3.091 & 3.097 \\
& $2\left(1^{--}\right)$ & ${ }^{3} S_{1}+{ }^{3} D_{1}$ & 3.901 & 3.686 \\
& $3\left(1^{--}\right)$ & ${ }^{3} S_{1}+{ }^{3} D_{1}$ & 4.767 & 3.040 \\
$\chi_{c 0}$ & $0^{++}$ & ${ }^{3} P_{0}$ & 3.409 & 3.415 \\
$\chi_{c 1}$ & $1^{++}$ & ${ }^{3} P_{1}$ & 3.451 & 3.511 \\
$\chi_{c 2}$ & ${ }^{3} P_{2}+{ }^{3} F_{2}$ & 3.556 & 3.779 \\
$\eta_{c 2}$ & $2^{-+}$ & ${ }^{1} D_{2}$ & 3.364 & \\
$h_{c 1}$ & $2^{+-}$ & ${ }^{1} P_{1}$ & 3.891 & \\
$\psi_{2}$ & $2^{--} D_{2}$ & & \\
\hline \hline
\end{tabular}


TABLE II: Mass-spectrum of bottomonium in units of GeV. The experimental values are from Ref. [13].

\begin{tabular}{|c|c|c|c|c|}
\hline Meson & $\mathrm{J}^{\mathrm{PC}}$ & ${ }^{\mathrm{s}} \mathrm{L}_{\mathrm{J}}$ & Theory & Experiment \\
\hline \multirow[t]{3}{*}{$\bar{\Upsilon}$} & $1^{--}$ & ${ }^{3} S_{1}+{ }^{3} D_{1}$ & 9.706 & 9.460 \\
\hline & $2\left(1^{--}\right)$ & ${ }^{3} S_{1}+{ }^{3} D_{1}$ & 10.054 & 10.023 \\
\hline & $3\left(1^{--}\right)$ & ${ }^{3} S_{1}+{ }^{3} D_{1}$ & 10.408 & 10.355 \\
\hline \multirow[t]{2}{*}{$\chi_{b 0}$} & $0^{++}$ & ${ }^{3} P_{0}$ & 9.873 & 9.860 \\
\hline & $2\left(0^{++}\right)$ & ${ }^{3} P_{0}$ & 10.224 & 10.235 \\
\hline \multirow[t]{2}{*}{$\chi_{b 1}$} & $1^{++}$ & ${ }^{3} P_{1}$ & 9.877 & 9.892 \\
\hline & $2\left(1^{++}\right)$ & ${ }^{3} P_{1}$ & 10.226 & 10.255 \\
\hline \multirow[t]{2}{*}{$\chi_{b 2}$} & $2^{++}$ & ${ }^{3} P_{2}+{ }^{3} F_{2}$ & 9.885 & 9.913 \\
\hline & $2^{++}$ & ${ }^{3} P_{2}+{ }^{3} F_{2}$ & 10.234 & 10.270 \\
\hline$\eta_{b}$ & $0^{-+}$ & ${ }^{1} S_{0}$ & 9.683 & \\
\hline$\eta_{b 2}$ & $2^{-+}$ & ${ }^{1} D_{2}$ & 10.011 & \\
\hline$h_{b 1}$ & $1^{+-}$ & ${ }^{1} P_{1}$ & 9.846 & \\
\hline$\Upsilon_{2}$ & $2^{--}$ & ${ }^{3} D_{2}$ & 10.053 & \\
\hline
\end{tabular}

TABLE III: The function $F(\Gamma, \mathbf{p})$ defined in the text, for various matrices $\Gamma$.

\begin{tabular}{lr}
\hline \hline$\Gamma$ & $F(\Gamma, \mathbf{p})$ \\
\hline 1 & $\cos \phi(p)$ \\
$\gamma_{0}$ & 1 \\
$\gamma_{5}$ & 0 \\
$\gamma_{j}$ & $\sin \phi(p) \widehat{\mathrm{p}}_{j}$ \\
$\alpha_{j}$ & $i \sin \phi(p)(\widehat{\mathbf{p}} \times \boldsymbol{\sigma})_{j}$ \\
$\Sigma_{j}$ & $\sigma_{j}+(\cos \phi(p)-1)(\widehat{\mathbf{p}} \cdot \boldsymbol{\sigma}) \widehat{\mathrm{p}}_{j}$ \\
\hline \hline
\end{tabular}

TABLE IV: The function $G(\Gamma, \mathbf{p})$ defined in the text. The symbols $s, t, c$ and $d$ are a short notation for: $s \equiv \sin \phi(p)$, $c \equiv \cos \phi(p), t \equiv \phi^{\prime}(p) / 2$ and $d \equiv \phi^{\prime} / p+\phi^{\prime \prime} / 2$.

\begin{tabular}{cc}
\hline \hline$\Gamma$ & $G(\Gamma, \mathbf{p})$ \\
\hline 1 & $-c t^{2}-s d+(1-c) / p^{2}$ \\
$\gamma_{0}$ & $-t^{2}+(c-1) / p^{2}$ \\
$\gamma_{5}$ & $\left(d-s / p^{2}\right)(\widehat{\mathbf{p}} \cdot \boldsymbol{\sigma})$ \\
$\gamma_{j}$ & {$\left[c d-s\left(t^{2}+1 / p^{2}\right)\right] \widehat{\mathbf{p}}_{j}+i\left(d-s / p^{2}\right)(\widehat{\mathbf{p}} \times \boldsymbol{\sigma})_{j}$} \\
$\alpha_{j}$ & $\left.-i\left[s\left(t^{2}+1 / p^{2}\right)-c d\right)\right](\widehat{\mathbf{p}} \times \boldsymbol{\sigma})_{j}+\left(d-s / p^{2}\right) \widehat{\mathrm{p}}_{j}$ \\
$\Sigma_{j}$ & $-\left(t^{2}+(1-c) / p^{2}\right) \sigma_{j}-\left[(c-1)\left(t^{2}+2 / p^{2}\right)+s d\right](\widehat{\mathbf{p}} \cdot \boldsymbol{\sigma}) \widehat{\mathrm{p}}_{j}$ \\
\hline \hline
\end{tabular}

TABLE V: The function $\mathbf{H}(\Gamma, \mathbf{p})$ defined in the text. The functions $s, t$ and $c$ have been defined as in table IV.

\begin{tabular}{cc}
\hline \hline$\Gamma$ & $\mathbf{H}(\Gamma, \mathbf{p})$ \\
\hline 1 & $i(1-c)(\widehat{\mathbf{p}} \times \boldsymbol{\sigma}) / 2 p-t s \widehat{\mathbf{p}}$ \\
$\gamma_{0}$ & $i(c-1)(\widehat{\mathbf{p}} \times \boldsymbol{\sigma}) / 2 p$ \\
$\gamma_{5}$ & $(t-s / 2 p)(\widehat{\mathbf{p}} \cdot \boldsymbol{\sigma}) \widehat{\mathbf{p}}+s \boldsymbol{\sigma} / 2 p$ \\
$\gamma_{j}$ & $(t c-s / 2 p) \widehat{\mathbf{p}}_{j} \widehat{\mathbf{p}}+s \sigma_{j} \boldsymbol{\sigma} / 2 p+i(t-s / 2 p)(\widehat{\mathbf{p}} \times \boldsymbol{\sigma})_{j} \widehat{\mathbf{p}}$ \\
$\alpha_{j}$ & $i(t c-s / 2 p)(\widehat{\mathbf{p}} \times \boldsymbol{\sigma})_{j} \widehat{\mathbf{p}}+s \sigma_{j} \boldsymbol{\sigma} / 2 p+(t-s / 2 p) \widehat{\mathrm{p}}_{j} \widehat{\mathbf{p}}$ \\
$\Sigma_{j}$ & $(c-1) \sigma_{j} \widehat{\mathbf{p}} / 2 p-(t s+(c-1) / p)(\widehat{\mathbf{p}} \cdot \boldsymbol{\sigma}) \widehat{\mathrm{p}}_{j} \widehat{\mathbf{p}}+(c-1)(\widehat{\mathbf{p}} \cdot \boldsymbol{\sigma}) \sigma_{j} \boldsymbol{\sigma} / 2 p$ \\
\hline \hline
\end{tabular}

